\title{
Formation of Large Target Residues in Intermediace Energy Nuclear Collisions
}

\author{
W. Loveland, $a$ K. A iek lett, $b$ L. Sihver, $b z, x u, a$ \\ and G.T. Seaborg
}

\author{
Nuclear Science Division \\ Lawrence Berkeley Laboratory \\ University of California \\ Berkeley, California 94720
}

\section{April 1987}

\begin{abstract}
DISCLAIMER
This report was prepared as an account of work sponsored by an agency of the United States Government. Neither the United States Government nor any agency thereof, nor any of their employees, makes any warranty, express or implied, or assumes any legal liability or responsibility for the accuracy, completeness, or usefulness of any information, apparatus, product, or process disclosed, or represents that its use would not infringe privately owned rights. Reference herein to any specific commercial product, process, or service by trade name, trademark, manufacturer, or otherwise does not necessarily constitute or imply its endorsement, recommendation, or favoring by the United Statcs Government or any agency thereof. The views and opinions of authors expressed herein do not necessarily state or reflect those of the United States Government or any agency thereof.
\end{abstract}

\section{a Oregon State University, Corvallis, Oregon 97331}

bThe Studsvik Neutron Research Laboratory, S-61182 Nyköping, Sweden

This work was supported by the Director, Office of Energy Research, Division of Nuclear Physics of the Office of High Energy and Nuclear Physics of the U.S. Department of Energy under Contract DE-AC03-76SF00098.

\section{MASTER}


W. LOVELANDa, K. ALEKLETTb, L. SIHVERb, Z.XUa, C. CASEYa, AND G.T. SEABORgC aOregon State University, Corvallis, OR 97331; bThe Studsvik Neutron Research Laboratory, S-61182 Nyköping. Sweden; CLawrence Berkeley Laboratory, Berkeley, CA $94720^{\star}$

We have used radiochemical techniques to measure the yields, angular distributions and velocity spectra of the large ( $A_{f r a g} \geq 2 / 3 A_{t g t}$ ) target residues from the fragmentation of $197_{\mathrm{Au}}$ by intermediate energy $12 \mathrm{C}, 20 \mathrm{Ne}, 32 \mathrm{~S}$, $40 \mathrm{Ar}, 84 \mathrm{Kr}$ and $139 \mathrm{La}$ projectiles. The fragrent moving frame angular distributions are asymmetric for the lighter projectiles (C-Ar). The fragment velocity spectra are Maxwellian for the $\mathrm{Kr}$ induced reactions and non-Maxwellian for the reactions induced by the lighter lons. We interpret these results in terms of a change in the dominant fragment production mechanism(s) from one(s) involving a fast non-equilibrium process for the lighter ions to a slow, equilibrium process for Kr. Comparison of the measured yields and angular distributions with calculations made using a Boltzmann transport equation with. appropriate modifications for Pauli blocking, etc. show excellent agreement between data and theory.

\section{INTRODUCTION}

Intemediate energy nucleus-nucleus collisions involving lighter (C-Ne) projectiles have been studied for several years. In this report, we focus our attention on the experimental characterization of intermediate energy nuclear collisions induced by heavier projectiles (S-La). Our emphasis will be on showing the changes in the character of these collisions as the projectile size, charge, etc. change. Our experimental focus wili be on measuring the properties of the large (Afragment $\geq 2 / 3$ Atarget) remnants ("evaporation residues") of the target nucleus left after such collisions. Our studies have been made using radiochemical activation techniques.

\section{EXPERIMENTAL}

The experiments to be discussed were carried out at the LBL 88" cyclotron (with its $17 \mathrm{~A} \mathrm{MeV} \mathrm{32S} \mathrm{beam).} \mathrm{the} \mathrm{MSU} \mathrm{superconducting} \mathrm{cyclotron} \mathrm{(32} \mathrm{A} \mathrm{MeV}$ $40 \mathrm{Ar}$ ), the GANIL accelerator complex ( $44 \mathrm{~A} \mathrm{MeV} 40 \mathrm{Ar}$ and $35 \mathrm{~A} \mathrm{MeV} 84 \mathrm{Kr}$ ) and the LBL Bevalac (150 A MeV 139La). Thin targets of $197_{\text {Au were irradiated }}$ by heavy ion beams with the evaporation residue and target fragment nuclei that recoil out of the target being collected in mylar catcher folls posi-

*Work sponsored in part by the U.S. Dept. of Energy under Contract DE-ACO376SF00098 and De-AM06-76RL02227 and the Swedish Natural Sciences Research Couneil. 
tioned on the walls of a cylindrical lucite chamber. Differential range spectra (which are converted to fragment velocity and energy spectra) were generally measured at three angles $\left(211^{\circ}, 90^{\circ}, 162^{\circ}\right)$ with respect to the incident beam. The angular distribution of the fragments was measured simultaneously as was the irradiation of a thicker target from which the absolute nuclidic yields (and ultimately the fragment isobaric yield distribution) were determined. The radioactivity of the trapped target recoils was measured by offline $r$-ray spectroscopy.

3. FRAGMENT MASS DISTRIBUTIONS

The fragment isobaric yield distributions from the interaction of various heavy jons with stmilar velocities with Au are shown in Figure 1.

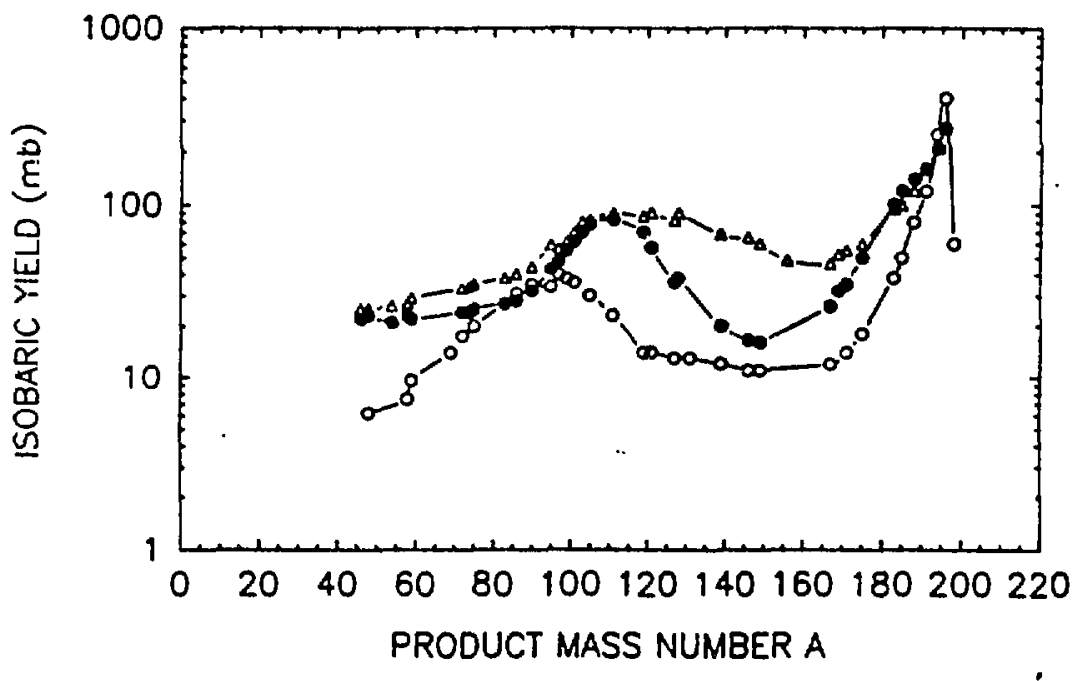

FIGURE 1

Target fragment isobaric yields from the interaction of $35 \mathrm{~A} \mathrm{MeV} 12 \mathrm{C}(-0-)$, $32 \mathrm{~A} \mathrm{MeV} 40 \mathrm{Ar}\left(-0_{-}\right)$and $35 \mathrm{~A} \mathrm{MEV} 84 \mathrm{Kr}(-\Delta-)$ with $\mathrm{Au}$. 
The yield distrfbution from the interaction of $35 \mathrm{~A} \mathrm{MeV} 12 \mathrm{C}$ with $\mathrm{Au}$ is characterized by a large bump at $A 295$ which is presumably due to the fission of a Au-like target residue. One can also observe a large yield of products with $A=160$ - 196 due (presumably) to the large remnants of incomplete fusion events. When the projectile stze is increased $(C \rightarrow A r)$, the central bump in the mass distribution shifts to larger mass numbers (Aน110) consistent with capture of a larger piece of the heavier projectile before fission. The mass distribution of the incomplete fusion residues extends to lower mass numbers and may contribute to the yields of events in the central bump (Section 3.). When the projectile size is further increased $(A r \rightarrow K r)$, the central bump in the mass distribution becomes quite broad and its centroid is at even larger mass numbers. (An120).

The mass distributions from the fragmentation of Au by higher energy ${ }^{12} \mathrm{C}$ and 139 La projectiles are quite different. (Figure 2).

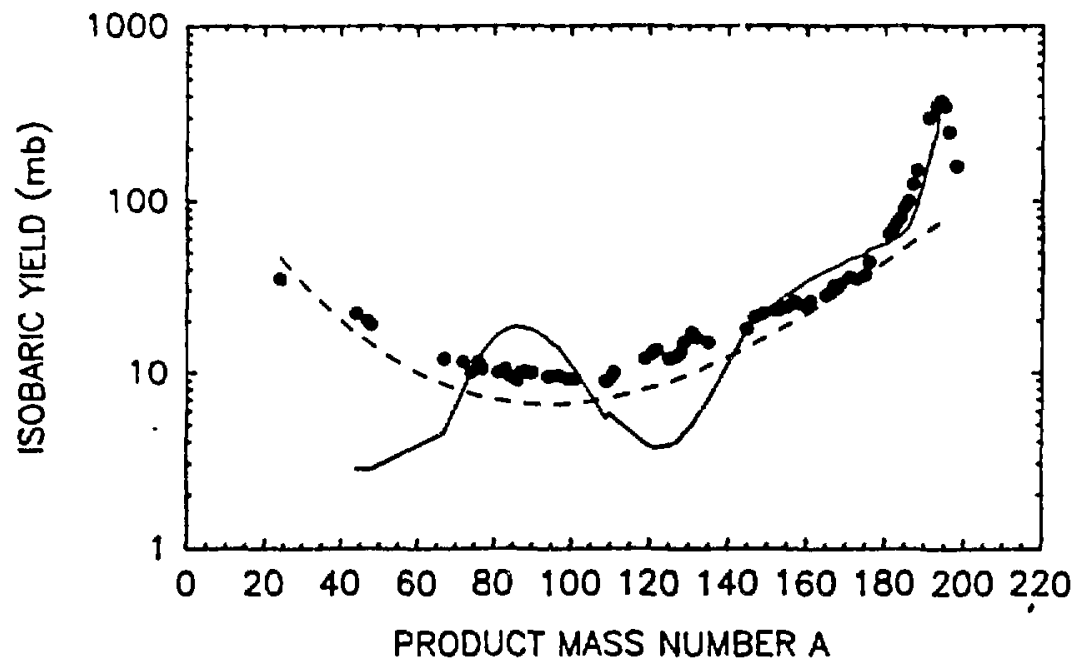

FIGURE 2

Fragment isobaric yield distributions for the interaction of $86 \mathrm{~A} \mathrm{MeV} 12 \mathrm{C}$ (solid line, ref. 1.), 400 A MeV 20 Ne (dashed line, ref. 2) and $150 \mathrm{~A} \mathrm{MeV}$ 139 La (0, this work) with $197_{\mathrm{Au}}$. 
Due to the increase in projectile size and energy, the distribution in the La induced reaction is quite dissimilar to that observed for the $C$ induced reactionl. The mass distribution for the $150 \mathrm{~A} \mathrm{MeV} \mathrm{La} \mathrm{induced} \mathrm{reaction} \mathrm{has}$ a similar shape to that observed 2 for the fragmentation of $\mathrm{Au}$ by $400 \mathrm{AMeV}$ $20 \mathrm{Ne}$ apart from the large yields of species near the target mass number.

\section{FRAGMENT ANGULAR DISTRIBUTIONS}

We have shown previously 3 for the interaction of $85 \mathrm{~A} \mathrm{MeV} 12 \mathrm{C}$ with $197 \mathrm{Au}$ and $238 \mathrm{U}$ that the normal fission fragment angular distributions were symetric about $90^{\circ}$ in the moving frame. We conclude for these events that the fissioning species lives long enough that the statistical assumption concerning level densities is valid, i.e., a statistically large number of overlapping levels is populated so that interferences between them will cancel. In the same $85 \mathrm{~A} \mathrm{MeV} 12 \mathrm{C}$ induced reactions, the moving frame angular distributions of the intermediate mass fragments (Afragment $<\frac{1}{3} A_{\text {target}}$ ) and the heavy target residues (Afragment $>\frac{2}{3} A_{\text {target}}$ ) were asymmetric with respect to $90^{\circ}$ indicating their production in a "fast" process without the establishment of statistical equilibrium. Thus the fragment angular distribution is taken as a gross measure of the time scale of the reaction (the time for equilibration has been calculated 4 to be $<2-3 \times 10^{-23} \mathrm{sec}$.). Furthermore, it was shown that the moving frame angular distributions for typlcal intermediate mass and heavy fragments such as $46 \mathrm{Sc}$ and $146 \mathrm{Gd}$ were kinematic complements supporting the idea that the production of these fragments is generally a "fast" binary reaction involving a very asymetric fission.

In Figure 3 we show the measured laboratory frame and deduced noving frame angular distributions for some typical fragments from the interaction of $49 \mathrm{~A} \mathrm{MeV} 20 \mathrm{Ne}$ and the previously discussed $85 \mathrm{~A} \mathrm{MeV} \mathrm{12C} \mathrm{with} 197 \mathrm{Au}$.

The laboratory frame angular distributions were transformed into the moving frame of the target residue following the initial projectile-nucleus encounter. The parameter $n_{11}\left(=v_{11 / N}\right.$ ) used to make the transformation (where $v_{11}$ is the longitudinal velocity of the moving frame and $V$ is the velocity of the fragment in the moving frame) was determined in two ways (which gave the same answer within experimental uncertainties): (a) from moving source fits to measured fragment velocity spectra (b) from integrating the angular distributions to give $F$ and $B$, the fraction of fragments recoliting forward and backward from the target $\left(n_{11}=(F-B) /(F+B)\right)$. 

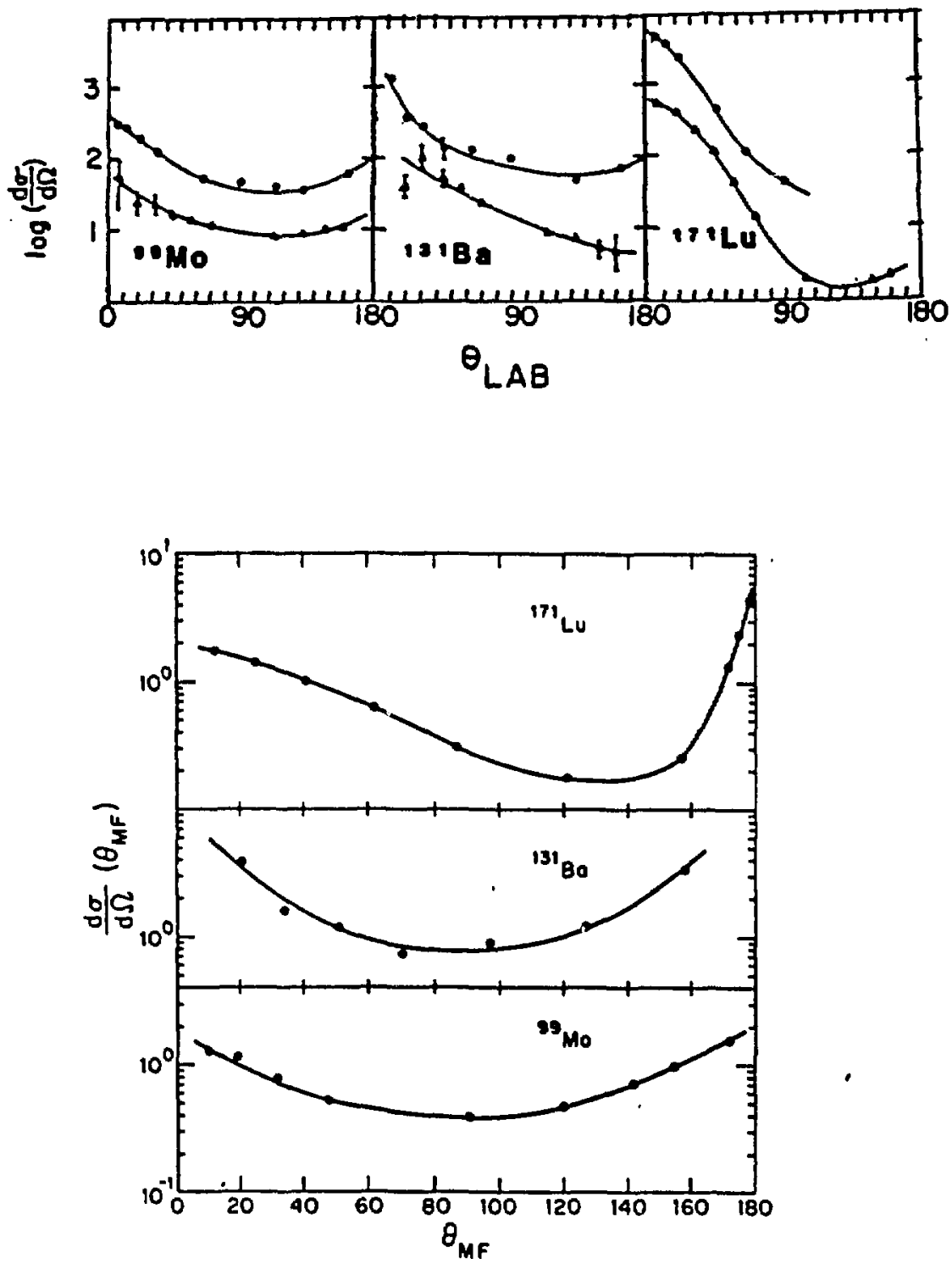

FIGURE 3

Typical laboratory frame and moving frame fragment angular distributions for the interaction of $49 \mathrm{~A} \mathrm{MeV} 20 \mathrm{Ne} \mathrm{(-)} \mathrm{and} 85 \mathrm{~A} \mathrm{MeV} \mathrm{12C} \mathrm{(4)} \mathrm{with} \mathrm{197} \mathrm{Au}$ 
Since there is a general similarity between the shapes of the laboratory frame angular distributions for the $12 \mathrm{C}$ and $20 \mathrm{Ne}$ induced reactions, it is not surprising that the moving frame angular distributions have simflar symetry properties. Moving frame distributions for normal fission fragments ( 99 Mo and $131 \mathrm{Ba}$ ) are symmetric with respect to $90^{\circ}$ indicating the occurrence of a "slow" process with the establishment of statistical equilibrium while the heavy fragment $\left(171_{L U}\right)$ angular distribution is asymetric in the moving frame indicating the production of these fragments in a "fast." non-equilibrium process.

A similar situation is observed when $A U$ is fragmented by a lower energy, larger heavy ion such as $17 \mathrm{~A} \mathrm{MeV} \mathrm{32s.} \mathrm{(Fig.} \mathrm{4)}$
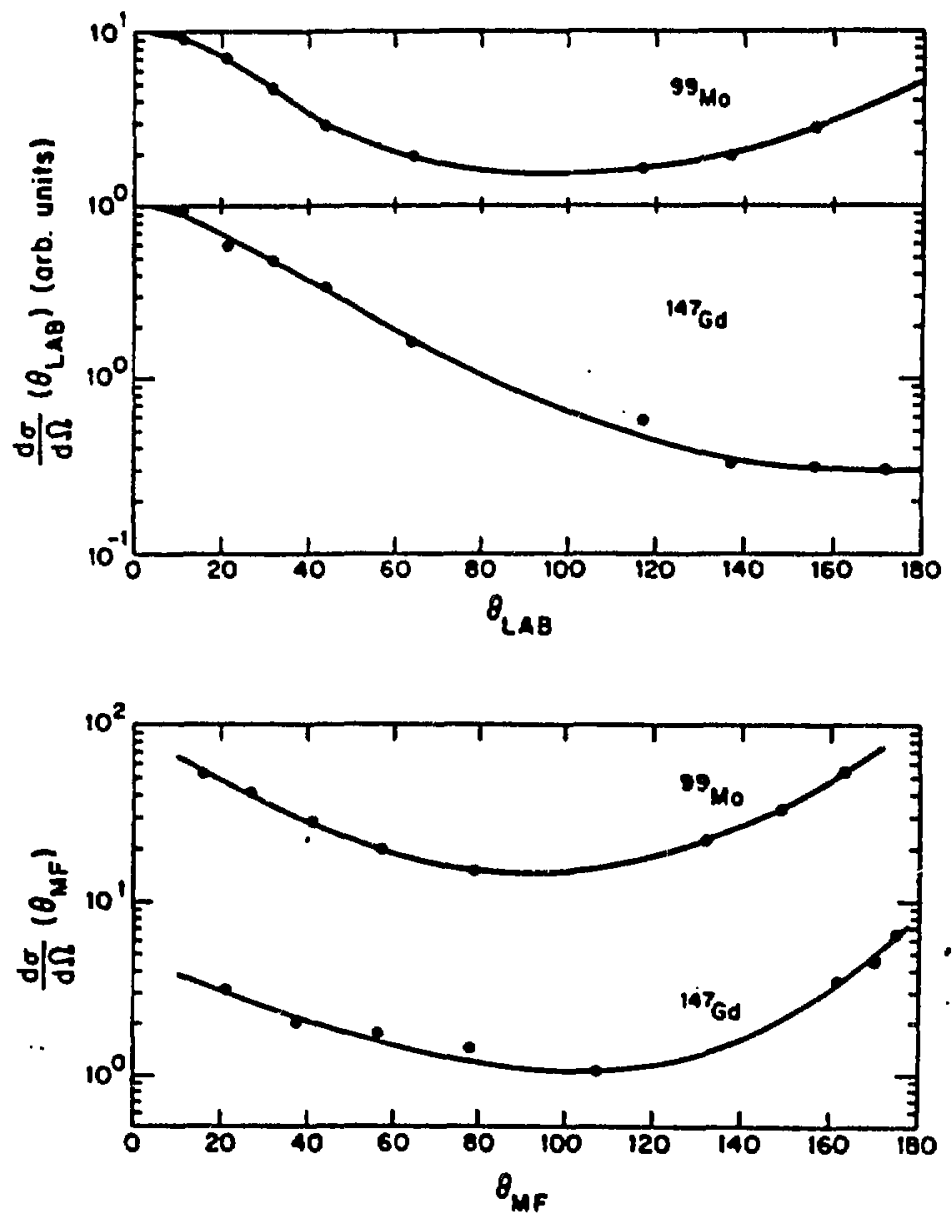

FIGURE 4

Laboratory frame and moving frame fragment angular distributions for the interaction of $17 \mathrm{~A} \mathrm{MeV} 32 \mathrm{~S}$ with $197 \mathrm{Au}$. 
Once again typical fission fragment $\left({ }^{99} \mathrm{MO}_{\mathrm{O}}\right)$ angular distributions are symmetric in the moving frame while those of the heavier fragments (such as $147 \mathrm{Gd}$ ) are not although we note that the 147 Gd is more nearly symmetric than heavy fragment distributions from reactions induced by lighter projectiles.

The laboratory frame angular distributions for the interaction of $32 \mathrm{~A}$ $\mathrm{MeV}$ and $44 \mathrm{~A} \mathrm{MeV} 40 \mathrm{Ar}$ with $197 \mathrm{Au}$ are similar. In the interests of brevity, we shall restrict our attention to the data from the higher energy reaction (Figure 5)
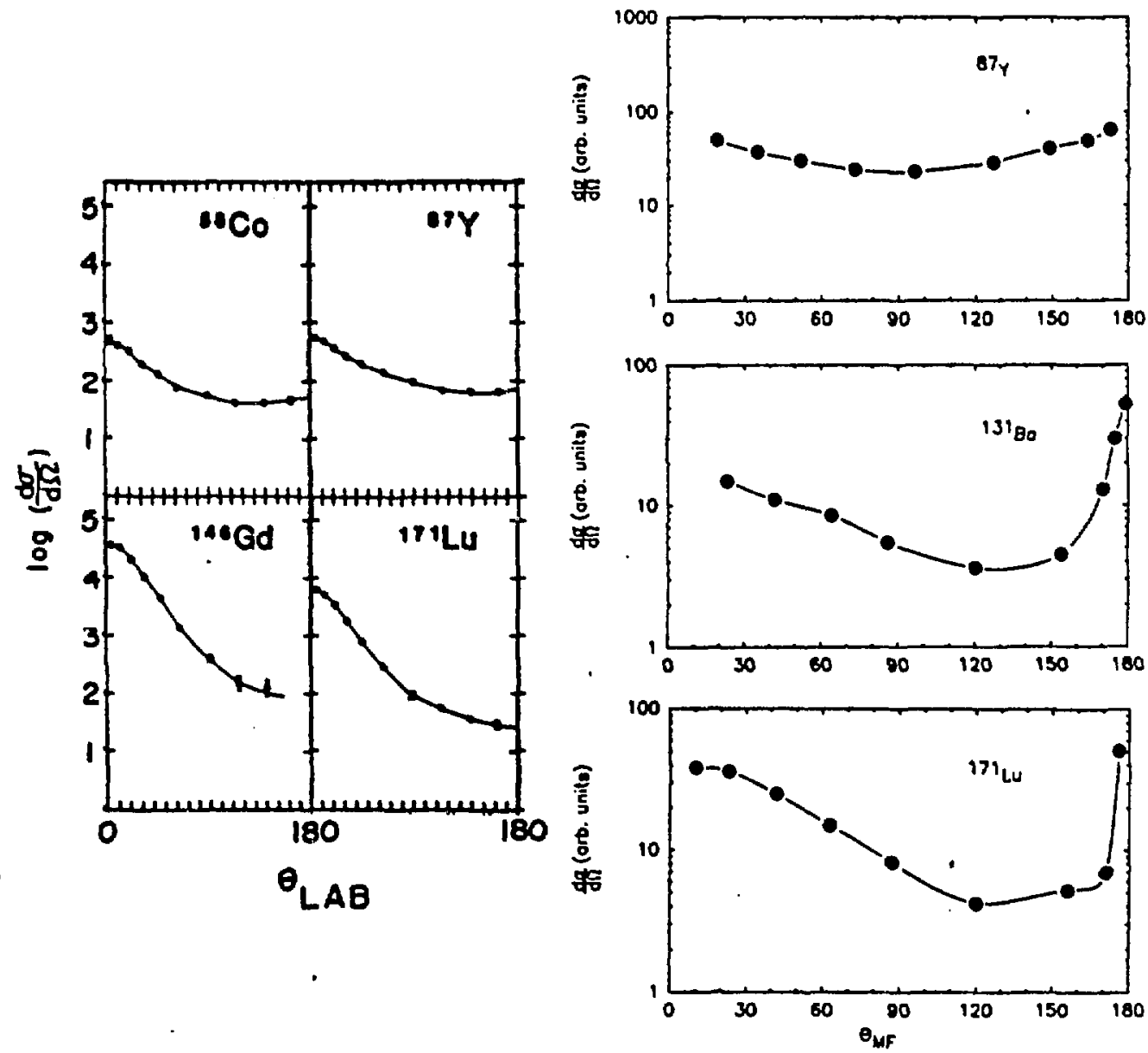

FIGURE 5

Typical laboratory and moving frame fragment angular distributions for the interaction of $44 \mathrm{~A} \mathrm{MeV} 40 \mathrm{Ar}$ with $197_{\mathrm{Au} \text {. }}$ 
The irving frame angular distribution for a typical fission fragment ( $87 \mathrm{Y}$ ) is symetric about $90^{\circ}$ while typical heavy members $\left(131_{\mathrm{Ba}}\right)$ of the central bump in the mass distribution (Fig. 1) have asymmetric angular distributions. This latter idea may be understood by the following argument. Assume the properties of "normal" fission are the same for the reactions of $49 \mathrm{~A} \mathrm{MeV}$ $20 \mathrm{Ne}$ and $44 \mathrm{~A} \mathrm{MeV} 40 \mathrm{Ar}$ with $\mathrm{Au}$ (Fig. 6). Subtract from the laboratory frame angular distribution of $131 \mathrm{Ba}$ from the $44 \mathrm{~A} \mathrm{MeV} 40 \mathrm{Ar}+\mathrm{Au}$ reaction the measured angular distribution from the $49 \mathrm{~A} \mathrm{MeV} 20 \mathrm{Ne}+\mathrm{Au}$ reaction (normalized at the most backward angle). The resulting distribution (Fig. 6) is very strongly forward peaked and presumably represents the contribution of a "fast", direct reaction to the production of $131 \mathrm{Ba}$ and similar high A members of the central bump in the isobaric yield distribution.
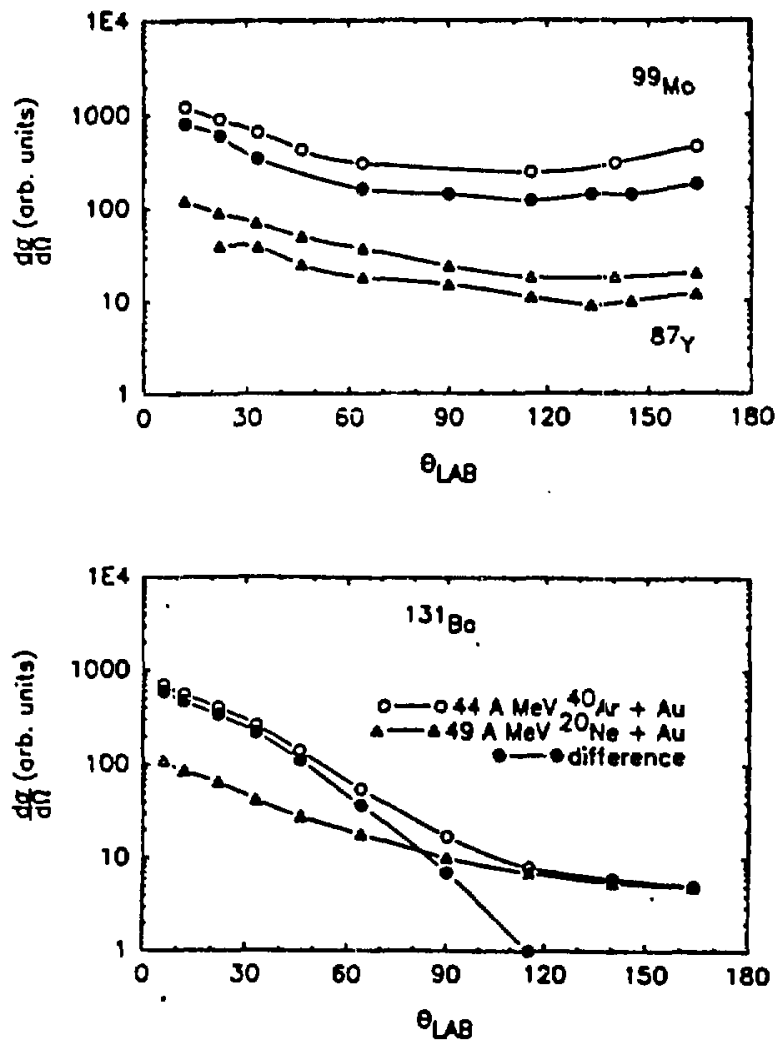

FIGURE 6

(a) Comparison of typical fission fragment angular distributions from the interaction of $49 \mathrm{~A} \mathrm{MeV} 20 \mathrm{Ne}$ and $44 \mathrm{~A} \mathrm{MeV} 4 \mathrm{CAr}_{\mathrm{Ar}}$ with $197 \mathrm{Au}$. Open sumbols

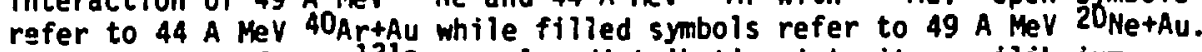

(b) Decomposition of the $131_{\mathrm{Ba}}$ angular distribution into its equilibrium and non-equilibrium components. 
The laboratory and moving frame angular distributions for the heavy fragments from the Interaction of $35 \mathrm{~A} \mathrm{MeV} 84 \mathrm{Kr}$ with $197_{\mathrm{Au}}$ (Fig.8) are qualitatively dissimilar to the distributions for similar fragments from reactions induced by the lighter ions. For the first time in our experience, the moving frame distributions of the heavy fragments are symmetric with respect to $90^{\circ}$, indicating the establishment of statistical equilibrium in the production of these fragments. Thus we are presented with a picture of an evolution in reaction mechanisms as we go from $12 \mathrm{C}$ induced reactions to $84 \mathrm{Kr}$ induced reactions with "fast" non-equilibrium processes dominating in the ${ }^{12} \mathrm{C}$ induced reactions while processes leading to the establishment of statistical equilibrium occur in the $84 \mathrm{Kr}$ induced reactions.

\section{FRAGMENT ENERGY SPECTRA}

Using fragment angular distributions as a guide, we focus our attention when examining the fragment energy spectra upon looking for evidence for a gradual establishment of equilibrium in the heavy fragment spectra as the projectile size increases. Alder and Wainwright have shown 5 that if one takes a group of hard spheres distributed uniformly in a volume $V$ with the same speed but moving in random directions, one can calculate the evolution of the velocity distribution in time (Figure 7 ).

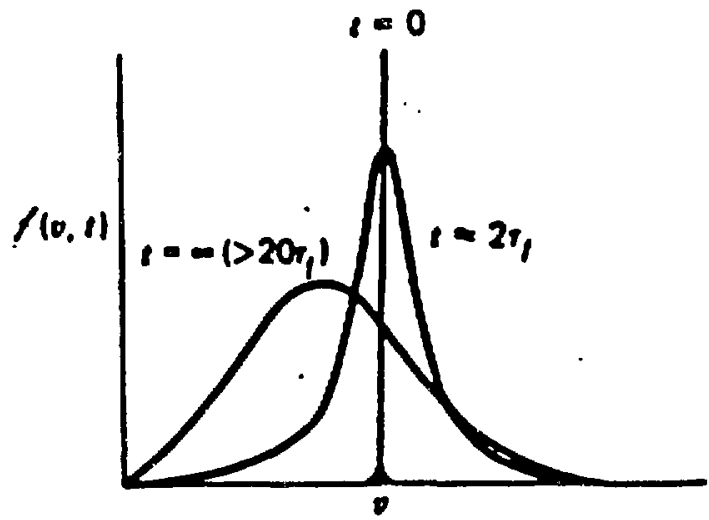

FIGURE 7

Calculated distribution of speeds as a function of time; if is the average time between collisions. 

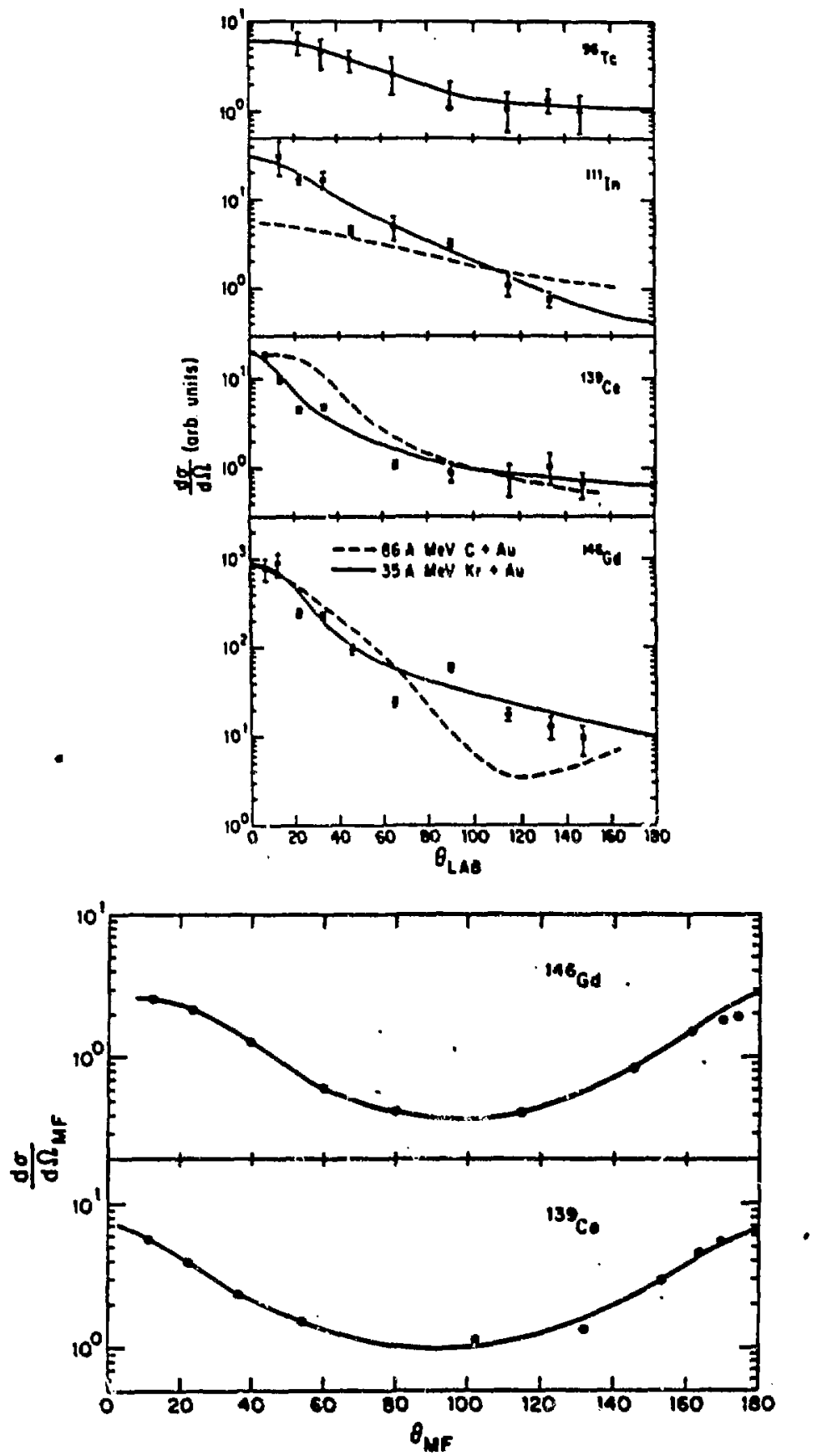

FIGURE 8

Laboratory and moving frame fragiant angular distributions from the fragmentation of Au by $35 \mathrm{MeV}^{84} \mathrm{Kr}$. 
One observes that the initial velocity distribution (assumed to be a spike) broadens and eventually evolves into the equilibrium Maxwell-Boltamann distribution after a modest number of collisions. If one further recalls that if a sphere (particle) escapes the volume, the resulting spheres (target remnant) will receive a momentum kick in the opposite direction equal to the momentum of the emitted particle. Thus the velocity (energy) distribution of target remnants might serve to sample the evolution of the reacting system towards statistical equilibrium.

With this idea in mind we can examine the heavy fragment energy spectra from the reaction of $85 \mathrm{~A} \mathrm{MeV} 12 \mathrm{C}$ and $35 \mathrm{~A} \mathrm{MeV} 84 \mathrm{Kr}$ with Au (Figure 9 ).

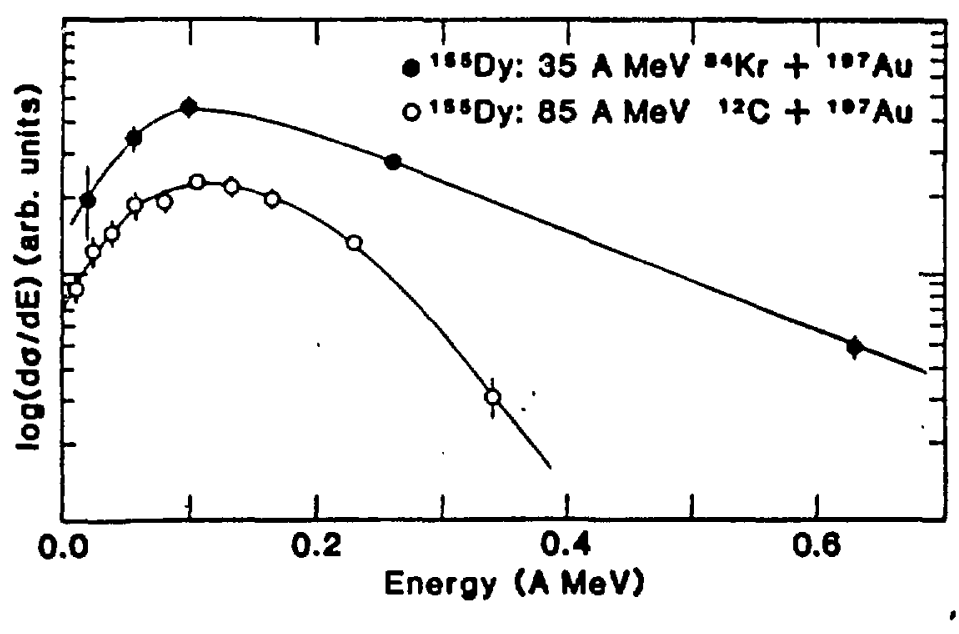

FIGURE 9

Forward angle energy spectra of heavy fragments from the fragmentation of Au by (a) $85 \mathrm{~A} \mathrm{MeV} 12 \mathrm{C}$ (b) $35 \mathrm{~A} \mathrm{MeV} 84 \mathrm{Kr}$. 
More detailed quantitative evaluation of these spectra shows that the spectra from the ${ }^{12} \mathrm{C}$ induced reaction do not have the equilibrium Maxwell-Boltzmann shape while those from the $84 \mathrm{Kr}$ induced reaction do. The extracted "slope parameters" or "pseudo-temperatures" of the latter spectra are $\sim 2.2-2.5 \mathrm{MeV}$. These observations are consistent with the trends observed in the angular distribution experiment.

\section{PRE-EQUILIBRIUM EMISSION CALCULATIONS}

Given the apparent importance of the evolution of the fragment angular and energy distributions towards equilibrium, we thought it would be appropriate to use the Boltzmann master equation model as developed by Blann 6 for use with intermecilate energy heavy ion reactions. In this model, the methods of quantum statistical mechanics are used to follow the propagation of excitation energy through the target nucleus. The target nucleus is assumed to be a two component Fermi gas with all states filled up to the Fermi level. Nucleons from the projectile are added to the target nucleus in states above the Fermi level. The relaxation of these initial configurations by internal nucleon-nucleon scattering or emission into the continuum is followed using a classical Boltzmann-like transport equation that has been appropriately modified to take into account quantum mechanical effects.

The basic Boltzmann-like transport equation has the form

$$
\begin{aligned}
\frac{d\left(N_{j} g_{i}\right)}{d t} & \left.=\sum_{j, k, l} w i j, k\right\}, i j g_{k} n_{k} g_{1} n_{1}\left(1-n_{j}\right)\left(1-n_{j}\right) g_{j} g_{j} \\
& -\sum_{j, k, l} w_{i j, k 1 g_{j} g_{j} n_{j}\left(1-n_{k}\right)\left(1-n_{k}\right)\left(1-n_{1}\right) g_{k} g_{1}-n_{i} g_{i} \omega_{1}, j^{-} g_{j}} \\
& +\frac{d}{d t}\left(n_{i} g_{j}\right) \text { fus, }
\end{aligned}
$$

where $n_{j}$ is the average occupation number and $g_{i}$ the number of single particle states per MeV in an energy interval 1 MeV wide measured from the bottom of the compound nucleus well. The wab,cd are the transition probabilities for nucleons in initial states a and $b$ to scatter into final states $c$ and d; they are evaluated from free nucleon-nucleon scattering cross sections. Pauli blocking is simulaied by the $\left(l-n_{j}\right)$ terms. The last term in equation (2) represents the time dependent injection of excitons into the fusing system. To actually perform these calculations we have used the computer code of Harp and Blann'. The only free parameter in this calculation was the initial exction number which was taken to be $\left(A_{p r o j}+3\right)$ for all cases. 
Using this model, we have calculated the average fractiolial linear momentum transfer to the target nucleus, and the spectra of the pre-equilibrium neutrons and protons for each system studied in this work. The calculated values of the fractional linear momentum transfer are in good agreement with systematics. To calculate the average angular momentum of the excited target residue, J, following the pre-equilibrium particle emission, we have used the simple semiclassical expression

$$
J=\frac{P_{11}}{P_{C N}} \cdot \ell_{C r i t}
$$

Values of $\ell_{c r i t}$ were taken from Ref. 8. The distribution of $\mathrm{J}$ values in the excited target residue was taken to be a $2 J+1$ distribution beginning at $\mathrm{J}=0$ and extending to $\mathrm{J}_{\max }$ with mean value $\mathrm{J}$. The distribution of excitation energy values in the excited residual nucleus $P\left(E^{*}\right)$ was computed using the calculated pre-equilibrium $n$ and $p$ kinetic energy spectra, the known binding energies and conservation of energy.

We assumed the excited target residue with excitation energy distribution $P\left(E^{\star}\right)$ and spin distribution $P(J)$ would de-excite to the final reaction products by equilibrium particle emission. This equilibrium particle emission was simulated using the computer codes PACE $^{9}$ and LINDA10. In calculating the angular distribution of the final product nuclei with the LINDA code, all the pre-equilibrium (direct) particles were assumed to be emitted at an angle.

$$
\theta=\frac{\hbar}{k R}
$$

where $R$ is the nuclear radius and $k$ the nucleon wave number. This assumption (krown as the Mantzourainis limitll) was made to be consistent with the calculations of the pre-equilibrium emission in the Blann model.

In Figure 10 we ccmpare the measured 12 and predicted isobartc yield distributions for the interaction of 8.5 and $19 \mathrm{MeV} /$ nucleon $16_{0}$ and $35 \mathrm{MeV} / \mathrm{nucleon}$ ${ }^{12} \mathrm{C}$ with $1545 \mathrm{~m}$. The agreement between, the measured isobaric ytelds and those predicted by the model is remarkably good, especially if one remembers the model has no adjustable parameters (as we used $i t$ ). The agreement between calculation and data extends over a change in the yields of two orders of magnitude, signifying a fundamentally correct simulation of the average behavior and the dispersion in that average. Good agreement is also obtained between the measured and calculated fragment angular distributions 12 . However, there are significant differences between the measured fragment velocity spectral2 for these reactions and these predicted by the Boltzmann master equation model (Figure 11 ). 
REPRODUCED FROM

BEST AVAILABLE COPY

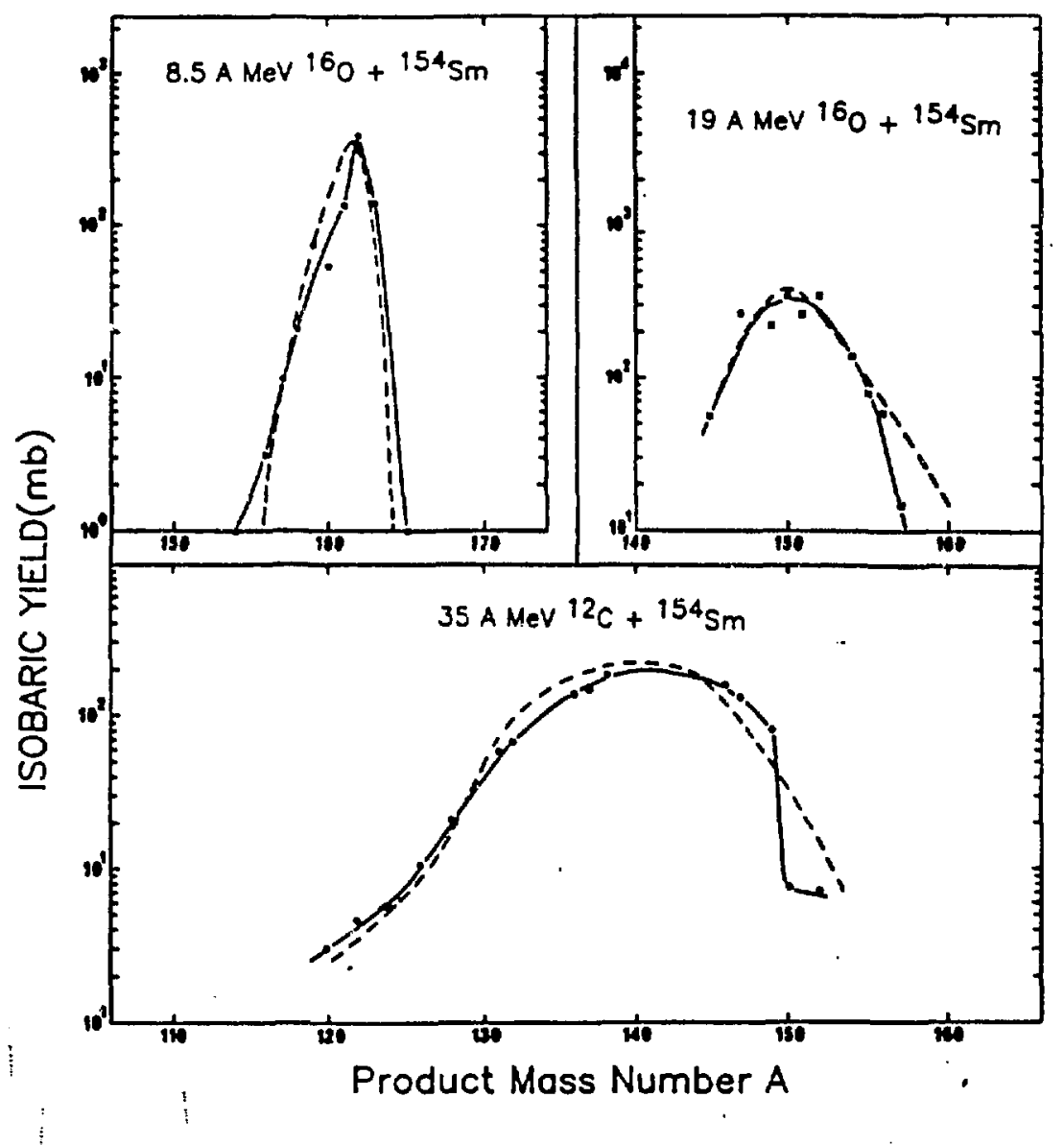

FIGURE 10

Comparison of measured isobaric yield distributions (solid line) with those predicted by the Boltzmann master equation model (dashed curve). 


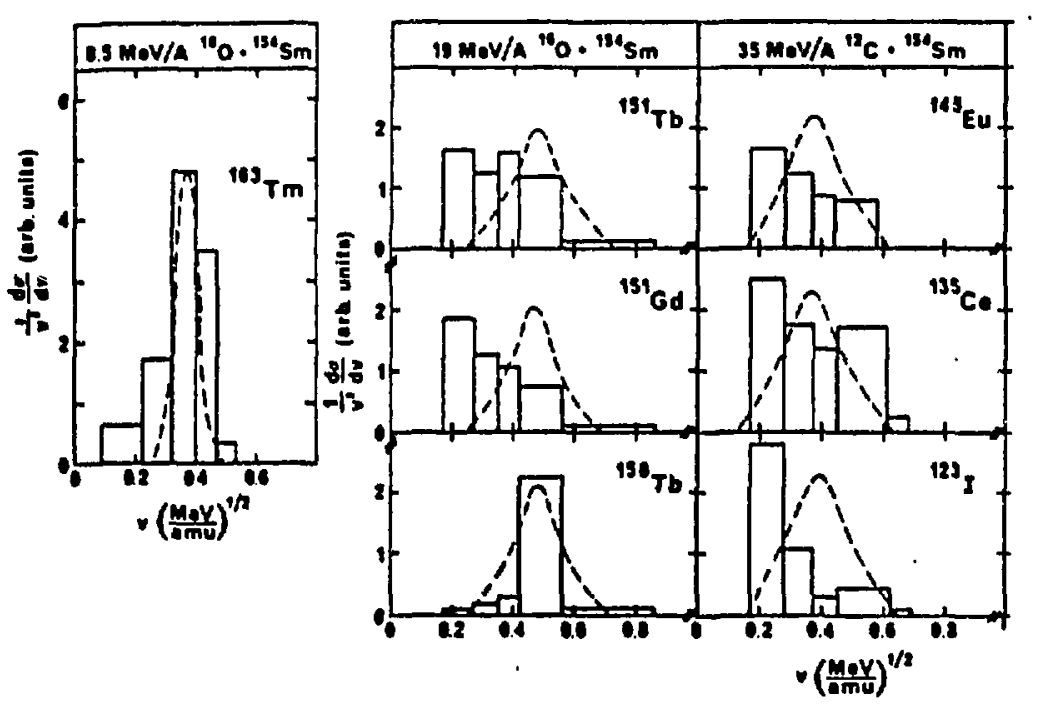

FIGURE 11

Measured (histograms) and predicted (dashed line) fragment velocity spectra. 
At the lowest projectile energy, $(8.5 \mathrm{MeV} / \mathrm{A} 160)$, the velocity spectra show Gaussian peaks centered near the velocity of the completely fused system (Figure 11). For the interaction of $8.5 \mathrm{MeV} / \mathrm{A} 160$ with $154 \mathrm{Sm}, 93 \%$ of the cross section is estimated to be assoctated with complete fusion reactions. The measured fragment velocity distribution for a typtcal fragment, ${ }^{163} \mathrm{Tm}$, agrees well with the predictions of the Boltzmann master equation model.

For the reaction of $19 \mathrm{MeV} / \mathrm{A} 16_{0}$ with $154 \mathrm{Sm}$, the velocity spectrum of 151 Tb represents that of a typical product. The velocity spectrum of $151 \mathrm{~Tb}$ can be thought of as a composite of spectra representing different reaction mechanisms, such as the spectra shown for $151_{\text {Gd }}$ (very incomplete fusion) and $156 \mathrm{~Tb}$ (near complete fusion). The average fragment velocity spectrum predicted by the Boltzmann master equation model is not in good agreement. with the data. The problem seems to be that whtle the model predfcts velocity spectra that have the correct shape and mean velocity for fusion-like processes (for example, production of $156 \mathrm{~Tb}$ ), the model fails to predict the changes in the shape of the velocity spectra when incomplete fuston processes occur (such as the production of $151_{\mathrm{Gd}}$ ). It should be noted that the model does correctly predict the overall average fragment velocity for the reaction.

For the reaction of $35 \mathrm{MeV} / \mathrm{A}{ }^{12} \mathrm{C}$ with $154 \mathrm{Sm}$, three typical fragment velocity spectra are shown. The nuclides involved represent different portions of the yield distribution for this reaction. All spectra show considerable amounts of incomplete fusion with mean fragment velocfties being $\sim 1 / 2$ that of the completely fused system. Because of the dominance of incomplete fusion processes in the velocity spectra of the representative fragments, the preequilibrium emission calcilations do not yieid velocity spectra with the correct shape although the mean fragment velocities are in agreement with the calculated results.

When we applied the Boltzmann master equation model to the fragmentation of Au as discussed in this paper, the failure of the model to correctiy treat incomplete fusion events proved to be serious (Figure 12). While the magnitude of the fission cross section is predicted correctly, the calculated yields of the heavy target residues are too low (due to the neglect of peripheral reactions).

\section{CONCLUSIONS}

We have shown how the isobaric yield distributions of target fragments vary as the projectile size increases from $C+L a$. Both the fragment angular distributions and energy spectra are consistent with a change in the dominant fragment production mechantsm(s) from one(s) involving a fast, non-equilibrium 
process for the lighter ions to a slow, equilibrium process for $\mathrm{Kr}$. Some success was achieved in describing data of this type using a Boltzmann master equation model of pre-equilibrium emission although the neglect of peripheral reactions in the model prevented a meaningful comparison of the model with our data.
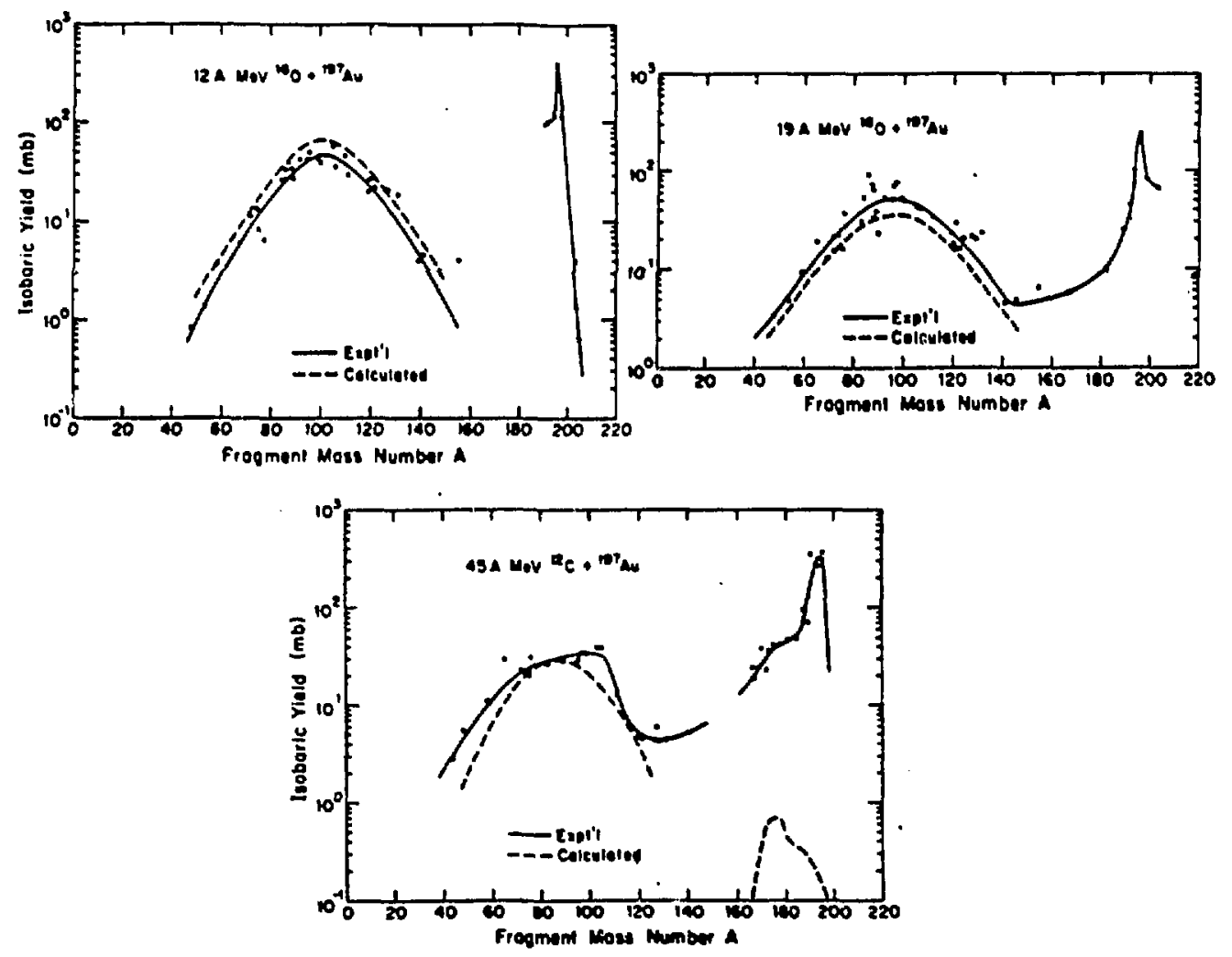

FIGURE 12

Measuredl (solid line) and predicted (dashed line) isobaric yield distributions for Au target fragmentation.

\section{ACKNONLEDGEMENTS}

We would like to gratefully acknowledge the assistance of D.J. Morrissey, M. de St. Simon and J.0. Liljenzin who participated in the setup and data collection phases of some of the experiments described herein.

\section{REFERENCES}

1) H. Loveland et al. . Lawrence Berkeley Laboratory report LBL-16280.

2) D.J. Morrissey et al. , Phys. Rev. C21 (1980) 1783. 
3) R.H. Kraus et al., Nucl. Phys. A432 (1985) 525.

K. Aleklett et al., Phys. Rev. $\underline{\text { C33 }}$ (1986) 885.

4) G.D. Harp, J.M. Miller and B.J. Berne, Phys. Rev. 165 (1968) 1166.

5) For a description of this work, see R.S. Berry, S.A. Rice and J. Ross, Physical Chemistry. (Wiley, New York, 1980) p. 1050.

6) M. Blann, Phys. Rev. C31 (1985) 1245.

7) M. Blann, Phys. Rev. C23 (1981) 205.

8) W.W. Wilke, J.R. Birkelund, H.J. Hollersheim, A.D. Hoover, J.R. Huizenga, W.U. Schroder and L.E. Tubbs, At. Data and Nucl. Data Tables 25 (1980).

9) A. Garron. Phys. Rev. C21 (1980) 230.

10) E. Duek, L. Kowalski and J.M. Alexander, Comp. Phys. Comm. 34 (1983) 395.

11) G. Mantzourainis, H.A. Heidenmiller, and D. Agassi, Z. Phys. A276 (1976) 145.

12) K. Aleklett, et al., Phys. Sci, 34 (1986) 489. 